Document downloaded from:

http://hdl.handle.net/10251/114606

This paper must be cited as:

Gómez Lozano, V.; Ramirez Hoyos, P.; Cervera, J.; Ali, M.; Nasir, S.; Ensinger, W.; Mafé, S. (2018). Concatenated logic functions using nanofluidic diodes with all-electrical inputs and outputs. Electrochemistry Communications. 88:52-56. doi:10.1016/j.elecom.2018.01.016

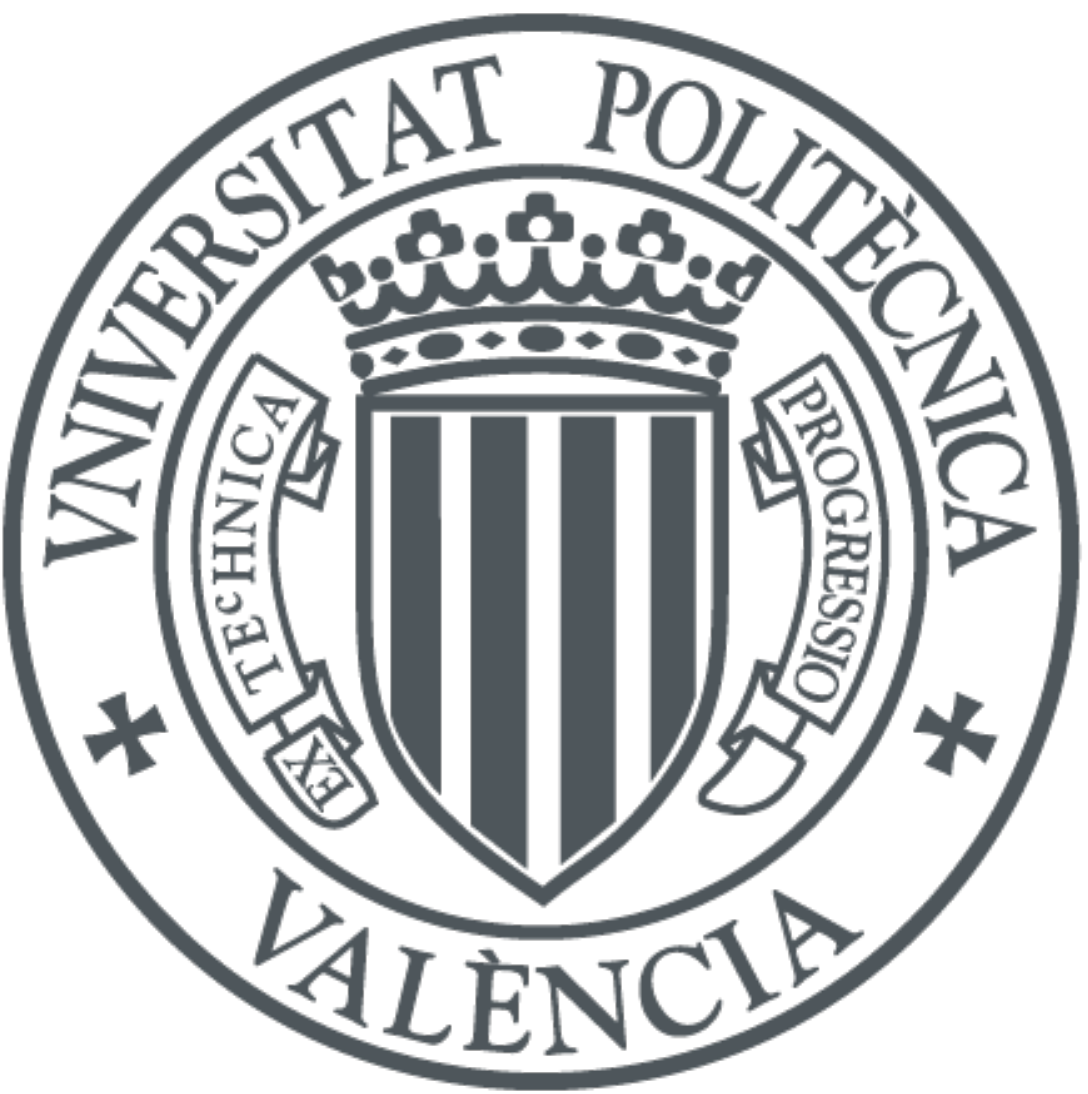

The final publication is available at

https://doi.org/10.1016/j.elecom.2018.01.016

Copyright Elsevier

Additional Information 


\section{Concatenated logic functions using nanofluidic diodes with all-electrical inputs and outputs}

Vicente Gomez ${ }^{\mathrm{a}}$, Patricio Ramirez ${ }^{\mathrm{a},{ }^{*}}$, Javier Cervera ${ }^{\mathrm{b}}$, Mubarak Ali ${ }^{\mathrm{c}, \mathrm{d}}$, Saima Nasir ${ }^{\mathrm{c}}$, Wolfgang Ensinger ${ }^{\mathrm{c}}$, Salvador Mafe ${ }^{\mathrm{b}}$

${ }^{a}$ Dept. de Física Aplicada. Univ. Politècnica de València. E-46022 Valencia, Spain

${ }^{b}$ Dept. de Física de la Terra i Termodinàmica, Universitat de València, E-46100 Burjassot, Spain

${ }^{c}$ Dept. of Material- and Geo-Sciences, Materials Analysis, Technische Universität Darmstadt, Petersenstr.23, D-64287 Darmstadt, Germany

${ }^{d}$ Materials Research Department, GSI Helmholtzzentrum für Schwerionenforschung, Planckstrasse 1, D-64291, Darmstadt, Germany

*Corresponding autor. Email address: patraho@fis.upv.es (P. Ramirez)

\section{ABSTRACT}

Nanopore-based logical schemes in ionic solutions typically involve single gates and chemical inputs. The design of computer-like functions requires the consecutive concatenation of several gates and the use of electrical potentials and currents to facilitate the downstream transfer of electrochemical information. We have demonstrated the robust operation of concatenated logic functions using biomimetic nanofluidic diodes based on single pore membranes. To this end, we have implemented first the logic functions AND and OR with combinations of single nanopores using all-electrical input and output signals. The concatenation of these gates allows the output of the OR gate to act as one of the inputs of the AND gate, giving an Enabled-OR logic function. Also, the operation of the OR gate connected with a solid-state transistor, working as a signal inverter, gives a NOR gate. These hybrid electrochemical circuits allow a variety of real time logic functions because of the robust electrical coupling between ionic solutions and electronic elements.

Keywords: nanofluidic diode, electrochemical logic functions, signal transduction. 


\section{Introduction}

Operational procedures with small electrochemical devices such as energy conversion modules, sensors and actuators, and controlled release drug-delivery dispensers require appropriate responses (outputs) to well-defined stimuli (inputs). This characteristic can be fulfilled by implementing logical schemes on micro and nanoscale devices such as selfassembled monolayers [1, 2], nanoparticle arrays [3, 4], and biomolecular systems [5]. Alternatively, micro and nanofluidic devices operating in ionic solutions permit logic functionalities by tuning the interaction between the molecules functionalized on the pore surface and free ions [6-15]. In particular, nanopore diodes inserted in polymeric membranes are responsive to chemical, electrical, thermal, and optical input signals, a crucial characteristic for sensors, energy conversion, and signal processing at bioelectrical interfaces [11, 16-23]. While the electronic technology dominates logic circuits, demonstrating electrochemically-based logical nanodevices allows information processing in the chemical and biological systems of biomedicine and biotechnology where soft matter micro and nanostructures operating in ionic aqueous environments are commonplace.

The charged inner walls of nanopores permit a variety of biomimetic responses in electrical rectification and switching processes $[12,17]$. Also, the possibility to interconnect these nanostructures with solid-state components and their compatibility with physiological fluids [19,24-28] can be exploited in devices allowing interactive communication with the human body. Most micro and nanopore-based logical schemes previously developed involve single gates and chemical (e.g., solution $\mathrm{pH}$ and analyte concentration) inputs [6,7,9,11-16]. However, sensing and actuating with combined chemical/electrical signals may require the design of logical functions of increased complexity for the downstream transfer of information in nanofluidic circuits. Once the first electrical output is acquired from the relevant chemical, thermal or optical input, signal transfer and processing can be implemented 
by concatenating a sequence of electrical signals in hybrid schemes combining nanofluidic and solid state circuitry. Indeed, the use of electrical signals such as potentials and currents should facilitate the sequential transfer of electrochemical information.

We consider here the design of concatenated logic functions using nanofluidic diodes with all-electrical input and output signals. To this end, we demonstrate first the logic functions OR and AND using combinations of single diodes. The concatenated operation of these gates, assembled in such a way that the output of the first gate constitutes one of the inputs of the second gate, allows an Enabled-OR function. Finally, we show that the operation of the OR gate connected with a solid-state transistor working as a signal inverter gives a universal NOR gate. The immobilization of the biomimetic nanopores on solid supports such as polymeric membranes should facilitate the sensing, switching, and resetting functions in different ionic solution environments.

\section{Experimental section}

Nanofluidic diode. The membrane samples containing single nanopores were obtained from stacks of $12.5-\mu \mathrm{m}$ thick polyimide (PI) foils (Kapton50 HN, DuPont) irradiated with swift heavy ions $(\mathrm{Au})$ of energy $11.4 \mathrm{MeV}$ per nucleon at the linear accelerator UNILAC (GSI, Darmstadt). In order to achieve single-ion irradiation, a metal mask with a $200 \mathrm{~mm}$ diameter centered aperture was placed in front of each stack. The ion beam was blocked immediately after a single ion passed through the foil stack and was registered by a particle detector placed behind the samples. The membrane tracks were converted into approximately conical pores by means of asymmetric track-etching techniques [29, 30]. SEM images of the nanopore fracture and gold replicas (see Fig. 1 for a typical image) of the conical pores can be found elsewhere [27]. Typical pore radii were in the range $10-40 \mathrm{~nm}$ for the cone tip and 300-600 $\mathrm{nm}$ for the cone base [27]. Because of the track-etching processes, carboxylate 
residues were obtained on the pore surface. These residues can be in ionized form, resulting in fixed charges, when the membrane is bathed by aqueous solutions of $\mathrm{KCl}$ at appropriate $\mathrm{pH}$ values $[7,9,17]$. These charges, together with approximately conical pore geometry, are responsible for the electrical rectification shown by the nanofluidic diodes $[9,17]$.

Electrical Measurements. Figs. 1-4 show the schemes of the equivalent electric circuits used for the AND, OR, Enabled-OR and NOR logic functions. The single pore membranes incorporating the nanofluidic diodes were bathed by $0.1 \mathrm{M} \mathrm{KCl}$ electrolyte solutions under controlled $\mathrm{pH}$ conditions. A picoammeter/voltage source (Keithley 6487/E) was used to introduce the input potential (square wave) $V_{1}$ and measure the electric current. Synchronized input signal waves $V_{2}$ and $V_{3}$ were obtained from $V_{1}$ using a MM74C93 4-bit binary counter and a 74HCT14 hex inverting Schmitt trigger. Output voltages were measured using a (Keithley 2000/E) multimeter. DC bias voltages were introduced in the circuits using a (Keithley 2400) source meter.

The input potentials and the resulting electric currents were introduced in the bathing solutions by $\mathrm{Ag} \mid \mathrm{AgCl}$ electrodes. Typical current-voltage $(I-V)$ curves showing the electrical rectification characteristics of the nanofluidic diodes can be found elsewhere $[7,9,17]$. The curves showed low pore resistances when the current entered the cone tip (positive potentials) and high resistances when the current entered the cone base (negative potentials) [31]. The PI samples used in the experiments showed currents of the order of $100 \mathrm{nA}$ for $\mathrm{V}=+2 \mathrm{~V}$ and -2 $\mathrm{nA}$ for $\mathrm{V}=-2 \mathrm{~V}$. The high rectification ratios obtained with these samples were needed in order to obtain robust logic responses in the output signals.

\section{Results and discussion}

The logic functions AND (Fig. 1) and OR (Fig. 2) are implemented by combinations of nanofluidic diodes with different polarities which are submitted to the time $(t)$-dependent 
input potentials $V_{1}$ and $V_{2}$. In each case, the potential $V_{\text {out }}$ is the output signal. Compared with the solid-state case, the logics " 0 " and " 1 " are only approximately defined because of the limited rectification characteristics of the nanofluidic diodes. However, the rectifying effects are significant and can be improved further by controlling the pore shape and charge distribution $[32,33]$.

Fig. 3 shows the scheme and operation of the concatenated logic obtained by connecting the above gates. The output of the OR gate is the first input for the AND gate. The integrated module can be operated as an Enabled-OR function because the input 1 of $V_{3}$ enables the logical function OR of the inputs $V_{1}$ and $V_{2}$. On the contrary, the input 0 of $V_{3}$ disables this function. The full availability of nanofluidic diodes showing different characteristics $[11,19$, 33], together with the possibility of using three-volume cells in the logical operation 14 allows the design of other concatenated gates with increased complexity.

Fig. 4 shows the operation of the OR gate of Fig. 2 when it is connected with a solidstate transistor to give a NOR universal gate. Note that any other logic function can be obtained as a combination of NOR gates only. The output of the OR gate acts as the input of the transistor working as a signal inverter. In order to correct for the mismatch between the electrical characteristics of the components of the hybrid circuit and achieve a high current gain, a Darlington pair is used. The electrical coupling between the two nanopores and the solid-state transistor allows integrating different device functionalities, as demonstrated recently for the case of nanopores and capacitors in energy conversion processes [28].

To achieve the real time modulation of Figs. 1-4, the externally applied input pulses should have time periods much larger than the relaxation time of the nanopore. Figs. 1-4 suggest that the time response of the nanofluidic circuits to the input signals is lower than $1 \mathrm{~s}$. This time may increase with the length $L$ of the pore, which is $L=10 \mu \mathrm{m}$ in our case. Using biological ion channels [34,35] and solid-state nanopores [18,19] fabricated on silicon oxide, 
ceramics, and glass supports, the response time of the system could be decreased because $L=$ $10-100 \mathrm{~nm}$ in these cases.

\section{Conclusions}

The implementation of processor-like functions using fluidic nanodevices requires the downstream transfer of electrochemical information. This essential characteristic can be achieved by concatenating several logic gates using different nanopore arrangements. We have described simple designs of concatenated logic functions using nanofluidic diodes with all-electrical input and output signals. We demonstrate first the logic functions OR and AND using different arrangements of single diodes and use then the output of the first gate as one of the inputs of the second gate to demonstrate an Enabled-OR logic function. The inverter functionality of a solid-state transistor connected to the output of an OR logic gate formed by two nanopores gives a universal NOR logics. The nanopores can be immobilized on solid single pore and multipore membranes that have previously been used for sensing and actuating. The resulting hybrid circuits allow a robust electrical coupling between ionic solutions and electronic elements such as transistors and capacitors.

\section{Acknowledgements}

P.R., J. C., and S. M. acknowledge the support from the Ministry of Economic Affairs and Competitiveness and FEDER (project MAT2015-65011-P). M.A., S.N. and W.E. acknowledge the funding from the Hessen State Ministry of Higher Education, Research and the Arts, Germany, under the LOEWE project iNAPO. 


\section{References}

[1] D.-H. Kim, H. Lee, C.-K. Song, C. Lee, Fabrication of nanosized molecular array device and logic gate using dimethyl-phenylethynyl thiol, J. Nanosci. Nanotechnol. 6 (2006) 34703473.

[2] J. A. Manzanares, J. Cervera, S. Mafe, Cooperative effects enhance electric-field-induced conductance switching in molecular monolayers, J. Phys. Chem. C 115 (2011) 6980-6985.

[3] K. Maeda, N. Okabayashi, S. Kano, S. Takeshita, D. Tanaka, M. Sakamoto, T. Teranishi, Y. Majima, Logic operations of chemically assembled single-electron transistor, ACS Nano 6 (2012) 2798-2803.

[4] J. Cervera, S. Mafe, Multivalued and reversible logic gates implemented with metallic nanoparticles and organic ligands, ChemPhysChem 11 (2010) 1654-1658.

[5] M. Pita, M. Krämer, J. Zhou, A. Poghossian, M. J. Schöning, V. M. Fernandez, E. Katz, Optoelectronic properties of nanostructured ensembles controlled by biomolecular logic systems, ACS Nano 2 (2008) 2160-2166.

[6] J.-H. Han, K. B. Kim, H. C. Kim, T. D. Chung, Ionic circuits based on polyelectrolyte diodes on a microchip, Angew. Chem. Int. Ed. 48 (2009) 3830-3833.

[7] M. Ali, S. Mafe, P. Ramirez, R. Neumann, W. Ensinger, Logic gates using nanofluidic diodes based on conical nanopores functionalized with polyprotic acid chains, Langmuir 25 (2009) 11993-11997.

[8] S. Mafe, J. A. Manzanares, P. Ramírez, Gating of nanopores: modeling and implementation of logic gates, J. Phys. Chem. C 114 (2010) 21287-21290.

[9] M. Ali, P. Ramirez, H. Q. Nguyen, S. Nasir, J. Cervera, S. Mafe, W. Ensinger, Single cigar-shaped nanopores functionalized with amphoteric amino acid chains: experimental and theoretical characterization, ACS Nano 6 (2012) 3631-3640. 
[10] M. Ali, S. Nasir, P. Ramirez, I. Ahmed, Q. H. Nguyen, L. Fruk, S. Mafe, W. Ensinger, Optical gating of photosensitive synthetic ion channels, Adv. Funct. Mat. 22 (2012) 390-396.

[11] P. Ramirez, M. Ali, W. Ensinger, S. Mafe, Information processing with a single multifunctional nanofluidic diode, Appl. Phys. Lett. 101 (2012) 133108.

[12] P. Ramirez, J. Cervera, M. Ali, W. Ensinger, S. Mafe, Logic functions with stimuliresponsive single nanopores, ChemElectroChem 1 (2014) 698-705.

[13] L. Zeng, Z. Yang, H. Zhang, X. Hou, Y. Tian, F. Yang, J. Zhou, L. Li, L. Jiang, Tunable ionic transport control inside a bio-inspired constructive bi-channel nanofluidic device, Small 10 (2014) 793-801.

[14] J. Cervera, P. Ramirez, V. Gomez, S. Nasir, M. Ali, W. Ensinger, P. Stroeve, S. Mafe, Multipore membranes with nanofluidic diodes allowing multifunctional rectification and logical responses, Appl. Phys. Lett. 108 (2016) 253701.

[15] H. Zhang, Y. Tian, L. Jiang, Fundamental studies and practical applications of bioinspired smart solid-state nanopores and nanochannels, Nano Today 11 (2016) 61-81.

[16] W. Guo, L. Cao, J. Xia, F.-Q. Nie, W. Ma, J. Xue, Y. Song, D. Zhu, Y. Wang, L. Jiang, Energy harvesting with single-ion-selective nanopores: a concentration-gradient-driven nanofluidic power source, Adv. Funct. Mater. 20 (2010) 1339-1344.

[17] Z. S. Siwy, S. Howorka, Engineered voltage-responsive nanopores, Chem. Soc. Rev. 39 (2010) 1115-1132.

[18] X. Duan, T.-M. Fu, J. Liu, C. M. Lieber, Nanoelectronics-biology frontier: from nanoscopic probes for action potential recording in live cells to three-dimensional cyborg tissues, Nano Today 8 (2013) 351-373.

[19] M. Tagliazucchi, I. Szleifer, Transport mechanisms in nanopores and nanochannels: can we mimic Nature?, Mater. Today 18 (2015) 131-142.

[20] H. Chun, T. D. Chung, Iontronics, Annu. Rev. Anal. Chem. 8 (2015) 441-462. 
[21] G. Pérez-Mitta, A. G. Albesa, C. Trautmann, M. E. Toimil-Molares, O. Azzaroni, Bioinspired integrated nanosystems based on solid-state nanopores: "Iontronic" transduction of biological, chemical and physical stimuli, Chem. Sci. 8 (2017) 890-913.

[22] P. Ramirez, V. Gomez, J. Cervera, S. Nasir, M. Ali, W. Ensinger, S. Mafe, Energy conversion from external fluctuating signals based on asymmetric nanopores, Nano Energy 16 (2015) 375-382.

[23] Y. Zhang, G. C. Schatz, Conical nanopores for efficient ion pumping and desalination, J. Phys. Chem. Lett., 8 (2017) 2842-2848.

[24] K. Healy, B. Schiedt, A. P. Morrison, Solid-state nanopore technologies for nanoporebased DNA analysis, Nanomedicine 2 (2007) 875-897.

[25] N. Misra, J. A. Martinez, S.-C. J. Huang, Y. Wang, P. Stroeve, C. P. Grigoropoulos, A. Noy, Bioelectronic silicon nanowire devices using functional membrane proteins, Proc. Natl. Acad. Sci. U.S.A. 106 (2009) 13780-13784.

[26] V. Gomez, P. Ramirez, J. Cervera, S. Nasir, M. Ali, W. Ensinger, S. Mafe, Charging a capacitor from an external fluctuating potential using a single conical nanopore, Sci. Rep. 5 (2015) 9501.

[27] P. Ramirez, V. Garcia-Morales, V. Gomez, M. Ali, S. Nasir, W. Ensinger, S. Mafe, Hybrid circuits with nanofluidic diodes and load capacitors, Phys. Rev. Applied 7 (2017) 064035.

[28] P. Ramirez, J.Cervera, V. Gomez, M. Ali, S. Nasir, W. Ensinger, S. Mafe, Optimizing energy transduction of fluctuating signals with nanofluidic diodes and load capacitors, Small (2018) doi: 10.1002/smll.201702252.

[29] P. Ramirez, P. Y. Apel, J. Cervera, S. Mafe, Pore structure and function of synthetic nanopores with fixed charges: tip shape and rectification properties, Nanotechnology 19 (2008) 315707. 
[30] W. Guo, Y. Tian, L. Jiang, Asymmetric ion transport through ion-channel-mimetic solidstate nanopores, Acc. Chem. Res. 46 (2013) 2834-2846.

[31] M. Queralt-Martin, E. Garcia-Gimenez, V. M. Aguilella, P. Ramirez, S. Mafe, A. Alcaraz, Electrical pumping of potassium ions against an external concentration gradient in a biological ion channel, Appl. Phys. Lett. 103 (2013) 043707.

[32] C. Verdia-Baguena, V. Gomez, J. Cervera, P. Ramirez, S. Mafe, Energy transduction and signal averaging of fluctuating electric fields by a single protein ion channel, Phys. Chem. Chem. Phys. 19 (2017) 292.

[33] Z. Siwy, I. D. Kosińska, A. Fuliński, C. R. Martin, Asymmetric diffusion through synthetic nanopores, Phys. Rev. Lett. 94 (2005) 048102.

[34] P. Apel, Track etching technique in membrane technology, Radiat. Meas. 34 (2001) 559566.

[35] J. Cervera, B. Schiedt, R. Neumann, S. Mafe, P. Ramirez, Ionic conduction, rectification, and selectivity in single conical nanopores, J. Chem. Phys. 124 (2006) 104706. 


\section{Figure captions}

\section{Fig. 1}

The logic function AND is achieved using nanofluidic diodes obtained by track-etching techniques. Typical gold replicas [27] are shown in the central image. The conical nanopores in single pore membranes are placed in an electrochemical cell and connected to a resistance $R=500 \mathrm{M} \Omega$ at potential $V_{0}=1.5 \mathrm{~V}$. The two square-wave input potentials $V_{1}$ and $V_{2}$ have different periodicity. The input potential $V_{3}$ allows the concatenation of this gate with other gates. The ground connection is also shown. A DC $3 \mathrm{~V}$ voltage is introduced in the circuit by means the long red and black wires. The potential $V_{\text {out }}$ is the output signal. The logics " 0 " and " 1 " correspond to low and high values of the input and output potentials, respectively.

\section{Fig. 2}

The logic function OR is realized by using two nanofluidic diodes with the ground resistance $R=500 \mathrm{M} \Omega$ and two square-wave input potentials. The input and output signals are those of Fig. 1.

\section{Fig. 3}

Scheme and operation of two concatenated OR and AND logic gates with $R_{1}=40 \mathrm{M} \Omega, R_{2}=$ $500 \mathrm{M} \Omega$, and $V_{0}=1.5 \mathrm{~V}$. These values of the resistances allow high electric currents in the first gate together with robust output potentials and logic responses in the second gate. The output of the OR gate corresponds to the input potentials $V_{1}$ and $V_{2}$ of Fig. 2 and acts as the first input for the AND gate to give an Enabled-OR logic function. The OR function is enabled only when the second input $\left(V_{3}\right)$ of the AND gate is 1 , while it is disabled when it is 0 . The system can be operated as an integrated module that allows the downstream transfer of information. 


\section{Fig. 4}

Scheme and operation of an OR logic gate connected to a solid-state transistor acting as an inverter. In order to achieve a significant current gain, a Darlington pair was used for the transistor, with $R_{1}=90 \mathrm{M} \Omega, R_{\mathrm{D}}=4.7 \mathrm{k} \Omega$, and $V_{\mathrm{D}}=3 \mathrm{~V}$. The resulting output is a universal NOR logic function. 


\section{AND logic function}

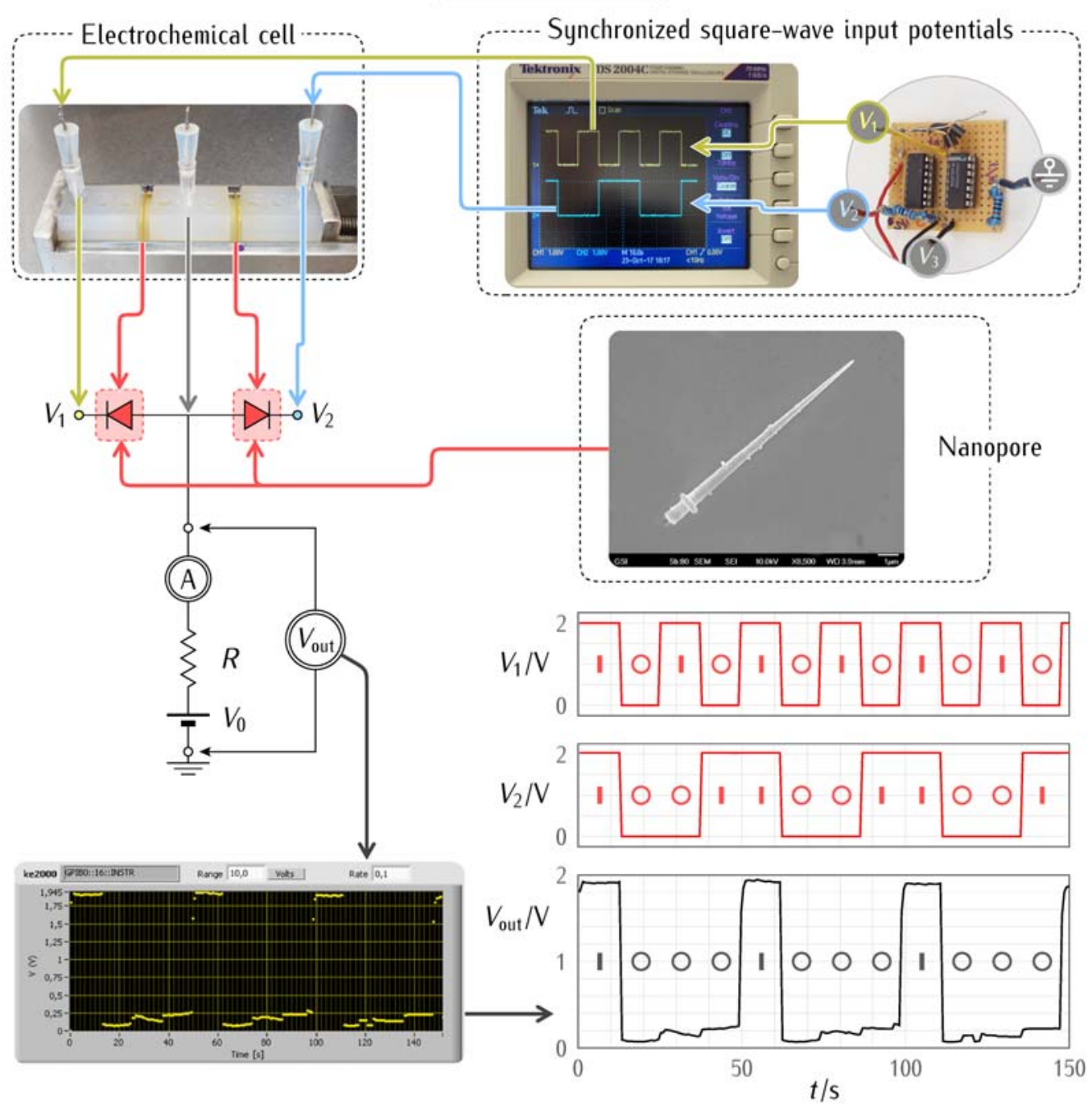

Figure 1 


\section{OR logic function}
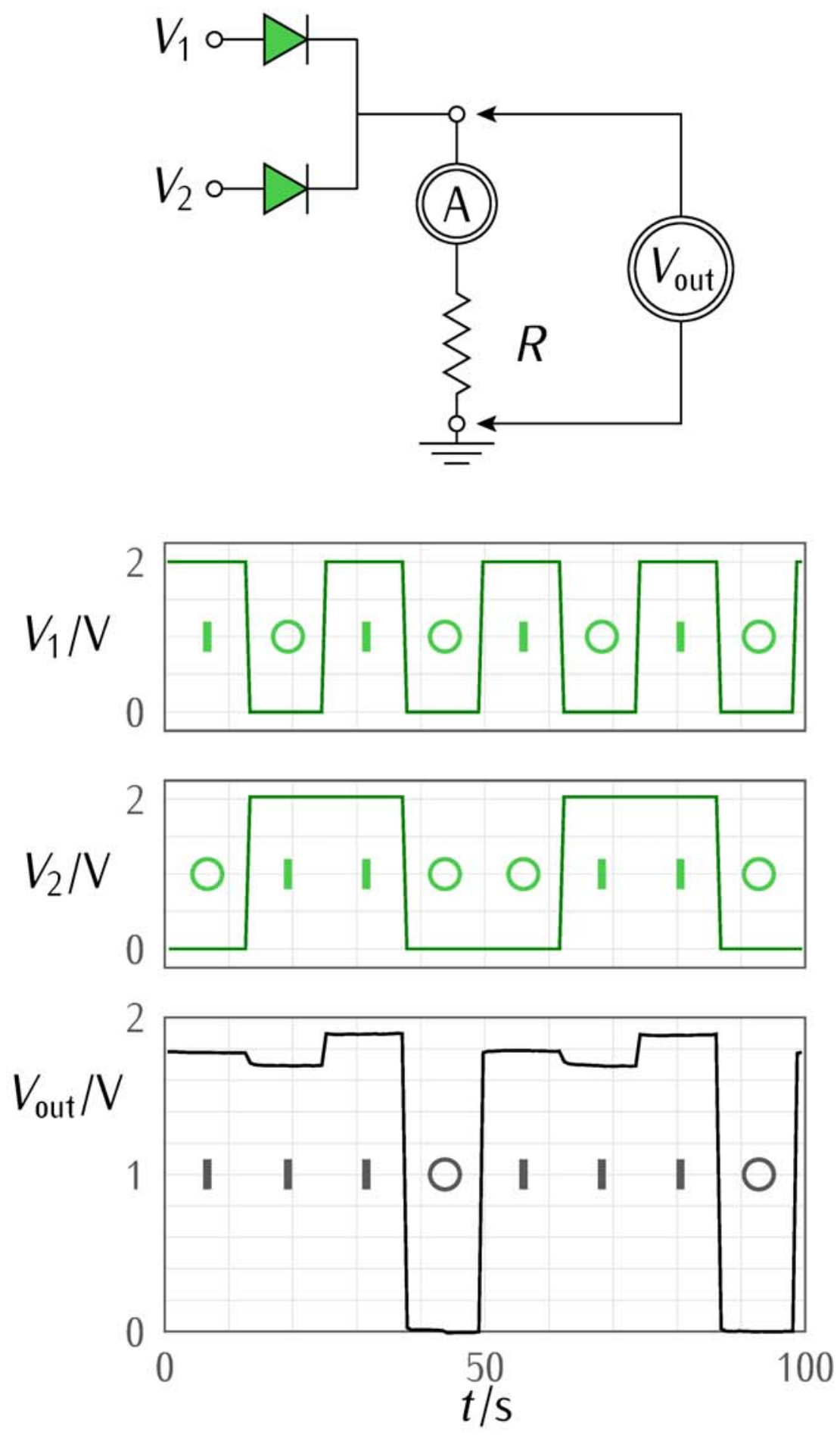

Figure 2 


\section{ENABLED-OR logic function}
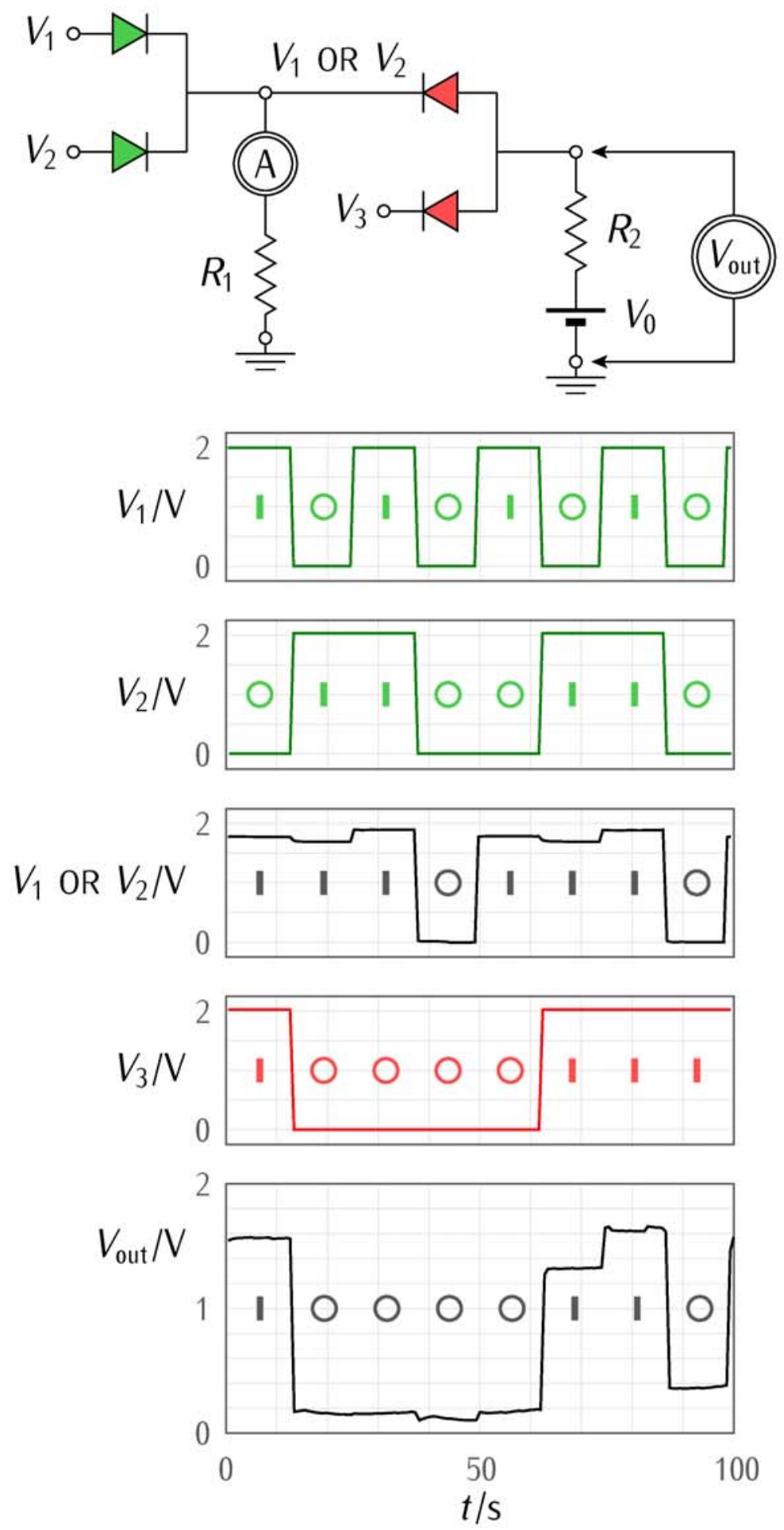

Figure 3 


\section{NOR logic function}
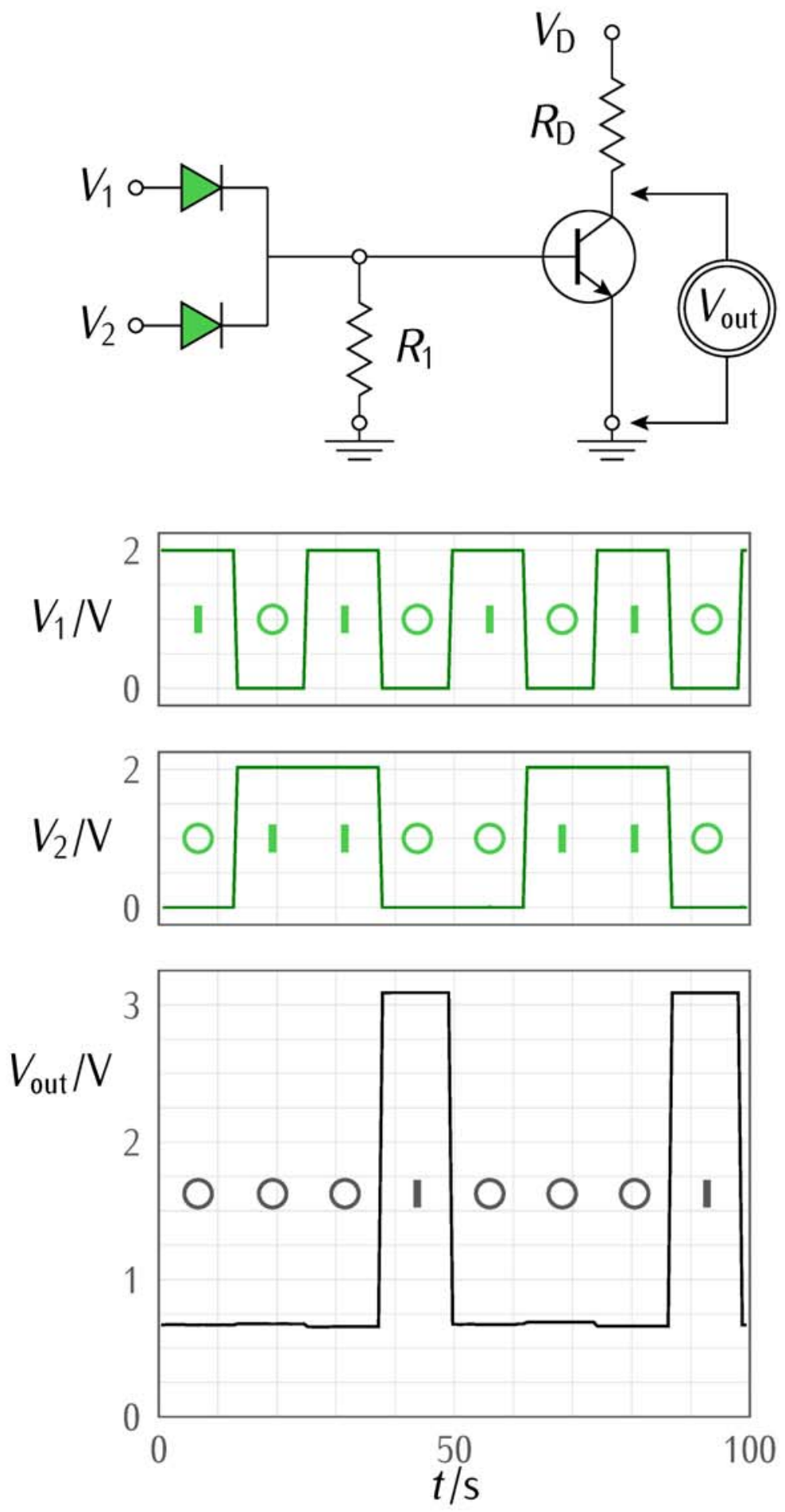

Figure 4 


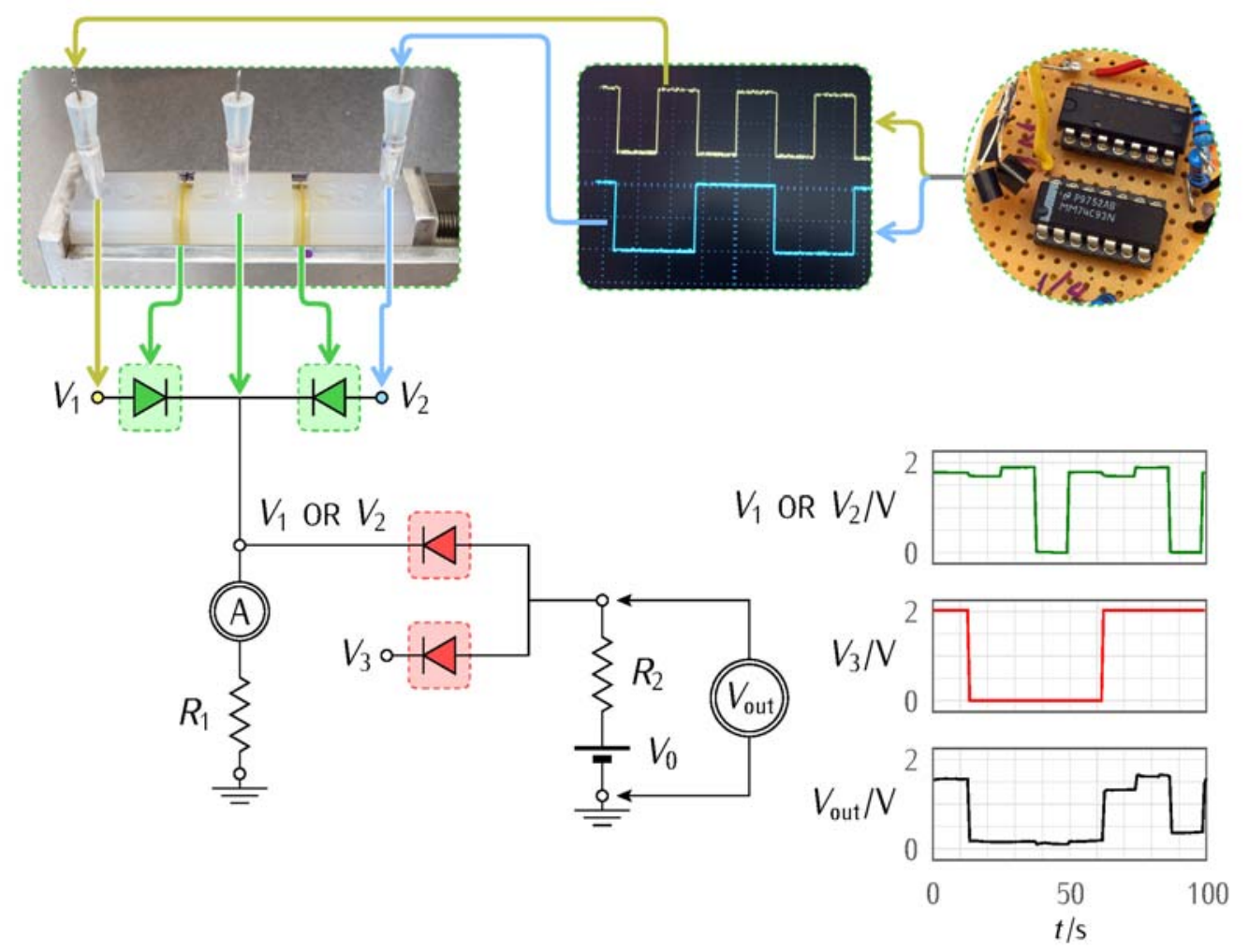

Graphical abstract 